\title{
ON A THEOREM OF IWASAWA
}

PAUL M. WEICHSEL

Iwasawa [1] has characterized those finite groups all of whose subgroups are either abelian, Hamiltonian, or nilpotent. He has shown that if the group order is divisible by more than two distinct primes then the group itself is either abelian, Hamiltonian, or nilpotent. The case of two primes has been treated by Miller [2] if all subgroups are abelian and by Iwasawa [1] if all subgroups are nilpotent.

In Theorem 1 a set of groups will be characterized which may be described by a property similar to that of Iwasawa: that every group contains abelian, Hamiltonian, or nilpotent $p$-complements for every prime dividing the order of the group. (A subgroup $H$ of $G$ is called a $p$-complement of $G$ if the index of $H$ in $G$ is equal to the order of a $p$-Sylow subgroup of $G$.) We first prove a lemma which then is generalized to apply to group properties which have inheritance characteristics similar to those of commutativity. In Theorem 2 we give an equivalent form of Theorem 1 with a somewhat more direct proof.

Lemma. ${ }^{1}$ Let the order of a group $G$, say $g$, be divisible by more than two distinct primes. If $G$ contains an abelian p-complement for all primes dividing the order of $G$ then every proper subgroup of $G$ is abelian.

Proof. Let $g=\prod_{i=1}^{L} p_{i}^{\alpha_{i}}$, all $\alpha_{i}$ are different from zero. Suppose $H$ is a proper subgroup of $G$ whose order is $h=\coprod_{i=1}^{L} p_{i}^{\beta_{i}}$. We first consider the case $\beta_{i}=0$ for some $i$. In this event $g=p_{i}^{\alpha_{i}} m$ with $(p, m)=1$. Now since $G$ contains a $p_{i}$-complement for all $i$, P. Hall $[3 ; 4]$ has shown that all subgroups of order $m$ are conjugate and every subgroup of $G$ whose order divides $m$ is contained in a subgroup of order $m$. Since there exists a subgroup of order $m$ which is abelian all subgroups of order $m$ are abelian. Hence in the case considered above $H$ is a subgroup of an abelian group and is hence abelian. Therefore we need only consider subgroups of $G$ whose order is divisible by all of the primes that divide $g$, i.e., $h=\prod_{i=1}^{L} p_{i}^{\beta_{i}}$ with $\beta_{i}$ different from zero for all $i$.

We now induct on the integer $\sum_{t=1}^{L} \beta_{i}$. The minimal case is $\sum_{i=1}^{L} \beta_{i}=L$. In this event all subgroups of $H$ are of the type treated above; that is, if $K \subset H$ then the order of $K$ is $\prod_{i=1}^{L} p_{i}^{\gamma_{i}}$ and $\gamma_{i}=0$

Received by the editors March 7, 1960.

1 This result reduces to the two prime case, the characterization solved by $\mathrm{C}$. Hobby, H. Rumsey, and the author in Finite groups having elements of every possible order, J. Washington Acad. Sci. vol. 50 (1960) pp. 11-12. 
for some $i$. Hence all subgroups of $H$ are abelian and since $p_{i} \mid h$ for all $i$, it follows from the Iwasawa theorem that $H$ is abelian. We therefore assume that all subgroups of $G$ for which $\sum_{l=1}^{L} \beta_{i} \leqq \theta$ are abelian. If there is a subgroup $K \subset G$ whose order is $\prod_{i=1}^{L} p_{i}^{\delta_{i}}$, with no $\delta_{i}=0$ and such that $\sum_{i=1}^{L} \delta_{i}=\theta+1$, then the order of every one of its subgroups is either not divisible by one of the prime divisors of $g$ or there is a subgroup of order $\prod_{i=1}^{L} p_{i}^{\epsilon_{i}}$, with no $\epsilon_{i}=0$, such that $\sum_{i=1}^{L} \epsilon_{i}<\theta+1$. In the first case the subgroup is abelian by the previous argument and in the second case it is abelian by the induction assumption. Hence all subgroups of $H$ are abelian and since $p_{i} \mid g$ implies $p_{i} \mid h, H$ is abelian by Iwasawa's theorem. This completes the proof of the lemma.

THEOREM 1. Let the order of a group $G$ be divisible by more than two distinct primes. $G$ is abelian if and only if for every prime dividing the order of $G, G$ contains a p-complement, which is abelian.

Proof. If $G$ contains an abelian $p$-complement for every prime dividing the order of $G$ then from the lemma all subgroups of $G$ are abelian. Hence by Iwasawa's theorem $G$ is abelian.

Conversely, if $G$ is abelian then it certainly contains all possible abelian $p$-complements.

Note that the lemma and theorem given above are equally valid if we substitute either Hamiltonian or nilpotent throughout. In fact the only property of abelian groups that we used in the proof of the lemma is that subgroups of abelian groups are abelian. Also the fact that the set of groups under consideration consists of those groups whose order is divisible by at least three primes was used in applying Iwasawa's theorem to those subgroups of $G$ whose order is divisible by all of the prime divisors of the order of $G$. We will therefore attempt to rephrase the theorem in greater generality.

Let $\Delta$ be a group theoretic property. We say that $G$ is a $\Delta$-group if $G$ has the property $\Delta$. Let $\Delta$ and a set of finite groups $\mathcal{G}$ be defined by:

I. If $G \in \mathcal{G}$ and $H \subseteq G$ then $H \in \mathcal{G}$ if the order of $H$ is divisible by the same primes which divide the order of $G$.

II. If a group $G$ is a $\Delta$-group then all subgroups of $G$ are $\Delta$-groups.

III. If $G \in \mathcal{G}$ then $G$ is a $\Delta$-group if all subgroups of $G$ are $\Delta$-groups.

We now assert

THEOREM A. If $G$ is soluble and $G \in \mathcal{G}$ then $G$ is a $\Delta$-group if and only if all p-complements of $G$ are $\Delta$-groups.

Proof. Since $G$ is soluble it contains all possible $p$-complements and the proof follows by substituting " $\Delta$ " for "abelian" and $\mathcal{G}$ as 
defined above for "the set of groups whose order is divisible by at least three primes" in the proof of the lemma.

We now complete the characterization of those groups which contain all possible $p$-complements and whose $p$-complements are either nilpotent, abelian or Hamiltonian. The only case not treated by Theorem 1 is that for which the order of $G, g=p^{\alpha} q^{\beta}$, with $p$ and $q$ distinct primes. Then the hypothesis of the lemma which requires that $G$ contain all possible $p$-complements is always satisfied. If we require such subgroups to be nilpotent then any group of order $p^{\alpha} q^{\beta}$ satisfies this condition. In the Hamiltonian case, one of the Sylow groups must be abelian since the only non-abelian Hamiltonian $p$ groups are 2-groups. Hence either both Sylow groups are abelian or else one of them is a direct product of an arbitrary abelian 2-group with the Quaternion group. Finally in the abelian case the condition is that the Sylow groups are abelian.

We now give a somewhat altered form of Theorem 1.

Theorem 2. A group $G$ of order $g$ is abelian, Hamiltonian, or nilpotent if and only if $G$ contains subgroups of order $p_{i}^{\alpha_{i}} p_{j}^{\alpha_{i}}$, for all $i, j$, with $g=\prod_{k=1}^{L} p_{k}^{\alpha_{k}}$ and these subgroups are abelian, Hamiltonian, or nilpotent.

Proof. We first consider the nilpotent case. Since there is a subgroup $H$ of order $p_{1}^{\alpha_{1}} p_{2}^{\alpha_{2}}$ and it is nilpotent it follows that the $p_{1^{-}}$ Sylow subgroup of $H, H_{1}$, permutes elementwise with some $p_{2}$-Sylow subgroup of $G$. But $G$ also contains a nilpotent subgroup $K$ of order $p_{1}^{\alpha_{1}} p_{3}^{\alpha_{3}}$. If the $p_{1}$-Sylow subgroup $K_{1}$ of $K$ is not equal to $H_{1}$, we replace $K$ by $x^{-1} K x$, where $x^{-1} K_{1} x=H_{1}$. Hence there is a $p_{i}$-Sylow subgroup for each $i=2, \cdots, L$ which permutes with a fixed $p_{1}$-Sylow subgroup, and therefore the $p_{1}$-Sylow subgroup is normal. A similar argument for the other primes yields the result that $G$ is nilpotent. If $G$ contains the required subgroups and they are abelian or Hamiltonian then they certainly are nilpotent and hence $G$ is nilpotent. But then $G$ is a direct product of either abelian or Hamiltonian groups and hence abelian or Hamiltonian. The converse is clear.

\section{REFERENCES}

1. K. Iwasawa, Über die Structur der endlichen Gruppen, deren echte Untergruppen sämtlich nilpotent sind, Proc. Phys.-Math. Soc. Japan vol. 23 (1941) pp. 1-4.

2. G. A. Miller and H. C. Moreno, Non-Abelian groups in which every subgroup is Abelian, Trans. Amer. Math. Soc. vol. 4 (1903) pp. 398-404.

3. P. Hall, A characteristic property of soluble groups, J. London Math. Soc. vol. 12 (1937) pp. 198-200.

4. - A note on soluble groups, J. London Math. Soc. vol. 3 (1928) pp. 98-105.

California Institute of Technology 